DIVISION OF THE HUMANITIES AND SOCIAL SCIENCES

CALIFORNIA INSTITUTE OF TECHNOLOGY

PASADENA, CALIFORNIA 91125

A REVEALED PREFERENCE APPROACH TO

COMPUTATIONAL COMPLEXITY IN ECONOMICS

Federico Echenique

Daniel Golovin

Adam Wierman

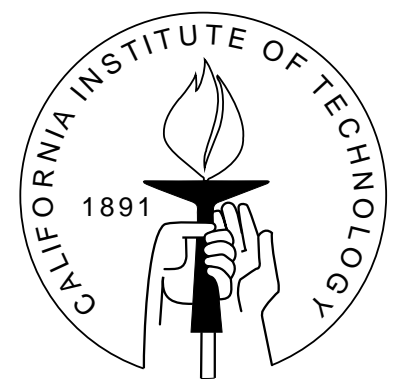

SOCIAL SCIENCE WORKING PAPER 1333 


\title{
A Revealed Preference Approach to Computational Complexity in Economics
}

\author{
Federico Echenique Daniel Golovin Adam Wierman
}

\begin{abstract}
One of the main building blocks of economics is the theory of the consumer, which postulates that consumers are utility maximizing. However, from a computational perspective, this model is called into question because the task of utility maximization subject to a budget constraint is computationally hard in the worst-case under reasonable assumptions. In this paper, we study the empirical consequences of strengthening consumer choice theory to enforce that utilities are computationally easy to maximize. We prove the possibly surprising result that computational constraints have no empirical consequences whatsoever for consumer choice theory. That is, a data set is consistent with a utility maximizing consumer if and only if a data set is consistent with a utility maximizing consumer having a utility function that can be maximized in strongly polynomial time.

Our result motivates a general approach for posing questions about the empirical content of computational constraints: the revealed preference approach to computational complexity. The approach complements the conventional worst-case view of computational complexity in important ways, and is methodologically close to mainstream economics.
\end{abstract}

JEL classification numbers: D01,C63

Key words: Computational Complexity, Strong Axiom of Revealed Preference, Revealed Preference, Theory of the consumer 


\title{
A Revealed Preference Approach to Computational Complexity in Economics*
}

\author{
Federico Echenique \\ Daniel Golovin \\ Adam Wierman
}

\section{Introduction}

Questions related to the computational complexity of economic models have been a driving force inspiring research at the intersection of computer science and economics. Over the last decade, many traditional economic models have been examined with the goal of understanding the computational requirements placed on the agents involved. For many models, this question has now been (partially) resolved.

For example computing Nash equilibria is now known to be hard $[12,14]$ even for 2player games [10], modulo reasonable complexity-theoretic assumptions ${ }^{1}$. Similarly, computing Walrasian equilibria in instances where agents have utilities that are separable over the goods and concave piecewise linear for each good is also hard [9, 35], specifically, PPAD-complete. On the positive side, it is known how to compute Walrasian equilibria in polynomial time if all agents have linear utility functions $[19,36]$. Still other results concern the complexity of the problems faced by individual agents within the market, such as a seller seeking profitmaximizing envy-free prices [18], or a seller seeking profit-maximizing prices when facing a collection of customers with known utility functions [3]. Both of those problems are NP-hard. From the buyers' perspective, problems as basic as maximizing utility under a budget constraint are NP-hard [17].

Computer scientists have often cited results such as those mentioned above as strong critiques of the applicability of classic economic models, claiming, for example:

"If an equilibrium is not efficiently computable, much of its credibility as a prediction of the behavior of rational agents is lost"

- Christos Papadimitriou [23]

${ }^{*}$ Echenique is affiliated with the Division of Humanities and Social Sciences; Golovin is affiliated with the Center for the Mathematics of Information at Caltech; Wierman is affiliated with Computers and Mathematical Sciences at Caltech. emails: fede, dgolovin, adamw@caltech.edu

${ }^{1}$ Computing 3-player Nash Equilibria is FIXP-complete [14]; the 2-player case is PPAD-complete [10]. These classes capture the difficulty of computing fixed points for large classes of functions. For additional background, we refer the interested reader to [11] and to [26]; the latter is specifically geared towards economists. 
"If your laptop cannot find it, neither can the market"

- quote attributed to Kamal Jain [23]

The critique amounts to a claim that either these computationally-hard models should be rejected, or that some suitable justification must be found for keeping them in spite of their worst-case hardness, e.g., by discovering some additional structure in real-world markets which makes converging to an equilibrium easy.

Economists have, in general, been slow to accept such critiques of these classic models. We posit that this is due to a differing view of the purpose of economic models, which leads to a contrasting notion of how computational issues should be incorporated. In particular, to an economist, models provide tools for thinking about reality; that is, about data. Thus, economic models/theories provide 'as-if' tools: economists postulate a model, and claim the observable variables behave as if the model were true. More specifically, given data from an observed phenomenon, we want to know if the theory can explain the data, i.e., if the data is consistent with an instance of the theory. If it is inconsistent, then the theory is refuted.

This differing view does not eliminate the need to consider computational issues in the model, it simply motivates a different approach for studying computational complexity. Specifically, the model still loses credibility if the agents must solve computationally hard problems; however, worst-case complexity is no longer the relevant concept. Instead, the question is whether data from an observed phenomenon can always be explained by the theory with the additional constraint that agents are not required to solve computationally hard problems, i.e., whether the data is consistent with an instance of the theory where the agents perform only computationally easy tasks. Note, of course, that this may be true even when the model is computationally hard in a worst-case sense.

This new perspective on computational complexity is strongly motivated by the revealed preference literature in economics [27]. This literature seeks to understand the empirical implications of economic models: given data from an observed phenomenon, the task is to understand how generally a model is applicable (i.e., how large the class of explainable data is) and to determine what instance of the theory is revealed by the data (e.g., to determine the properties of the utility functions that are consistent with the data). Examples of such results include $[1,2,32,33,22,6,5,13,7,8]$. Hal Varian [34] has an excellent survey of revealed preference.

Revealed preference theory, however, traditionally disregards computational issues (as do most economists, in general). Our proposed perspective on complexity changes this by adding to revealed preference theory the constraint that the instance revealed does not require agents to solve any computationally hard problems. Thus, the question becomes, does the addition of computational constraints limit the class of data explainable by the theory? Or, more colloquially, do computational constraints have empirical consequences for economic models?

The goal of this paper is to formalize this "revealed preference view" of computational complexity, and to begin to explore whether it leads to different conclusions than the standard "worst-case view." To this end, we study a classic economic model: consumer choice theory. 
Consumer choice theory involves ideas that are familiar and important to both the computer science and economics communities - the theory is the basic building block of economics. Its central postulate is that consumers are utility maximizing, and yet the very task of utility maximization can be seen to be NP-hard as an immediate consequence of classical results in computer science. For consumer choice theory, the case made above-that the theory is flawed because it assumes that agents solve hard problems - is very natural, and easy to understand. A recent presentation of this view of consumer choice theory is in [17]; but the case has been presented (albeit informally) many times before ([29]). ${ }^{2}$ In this paper, we shall present a different view, based on the revealed preference approach to consumer theory.

The specific model we consider (Section 2) is that of a single consumer who, when presented with a budget and prices for $d$ goods, must decide on a consumption bundle. It is not difficult to see that, in this model, utility-maximizing consumers must solve a generalization of the knapsack problem, and thus, in a worst-case sense, the model requires consumers to solve an NP-hard problem. However, when the model is considered using the "revealed preference view" of computational complexity the conclusion is different. We prove that, given data for the observed behavior of a consumer, the data either corresponds to irrational behavior (it cannot be reconciled with the optimization of any utility function) or it can be explained using tractable utility functions, i.e., those that are computationally easy to maximize subject to a budget constraint. Thus, if there exist utility functions that explain the data, then there exists a tractable utility function that explains the data. In other words, the constraint that the consumer's utility function is tractable does not alter the range of consumer behavior that may be explained by the theory. So, for this model, computational constraints have no empirical consequences-despite the fact that the underlying problems the agents must solve are NP-hard in the worst-case.

As an application of our results on consumer choice, we study Walrasian (competitive) equilibrium theory (Section 3). The specific model we consider is that of an exchange economy with $d$ goods and $m$ utility-maximizing agents, each having an endowment and a utility function. A simple extension to the results for the consumer choice model described above shows that: given a data set including observations of prices and allocations of goods to agents, either the data corresponds to behavior that is manifestly irrational or the data can be explained using utility functions that are computationally easy to maximize and that explain the market behavior. So, again, for this model, computational constraints have no empirical consequences. We should stress, however, that the results for Walrasian equilibrium do not address the computational complexity of finding an equilibrium. We only focus on the complexity of the agents' maximization problem.

In both economic models considered in this paper, agents must perform computations that are hard in the worst-case sense. An "economist's view," however, of computational complexity shows that computational constraints do not limit the applicability of the economic models any further than the assumption of rationality does already. This provides a stark contrast to the message from the worst-case computational complexity studies of these models.

\footnotetext{
${ }^{2}$ Herbert Simon's was one of the earliest voices for incorporating computational constraints in economic models. Simon was aware, however, that the complexity of the procedures involved in decision making would only matter to the extent that they made a difference empirically; see [29].
} 
We emphasize that this paper considers only two basic models, and an important goal for further research is to understand whether similar results hold for other classic economic models such as Nash equilibrium, or for the problem of finding an equilibrium in a competitive market. Toward this end, in Section 4, we present a general formulation of the revealed preference view of computational complexity which provides a framework for approaching the analysis of other economic theories.

\section{Consumer choice theory}

The revealed preference approach we propose is widely applicable, but consumer choice theory is a natural starting point since the theory is a basic building block for most of economics. It is the first topic covered in every introductory course in economics, and it remains a very active research field until this day: active for both empirical and theoretical research. Further, the revealed preference approach in economics was first proposed and developed for consumer choice theory.

Before introducing the model formally, we introduce some notation that will be used throughout the remainder of the paper. For integer $n$, we let $[n]:=\{1,2, \ldots, n\}$. Let $\mathbb{Z}_{+}$and $\mathbb{R}_{+}$denote the nonnegative integers and real numbers, respectively, and let $\mathbb{Z}_{++}$and $\mathbb{R}_{++}$and denote the set of positive integers and real numbers, respectively. For a binary relation $R$, we let $R^{*}$ denote its transitive closure. A binary relation $R$ is acyclic if $x R^{*} y$ and $y R^{*} x$ implies that $x=y$. All logarithms are base 2 unless otherwise noted. We denote a $d$-dimensional vector with all coordinates equal to $c$ by $\mathbf{c}$ and denote the $i^{\text {th }}$ basis vector, which is one at coordinate $i$ and zero at the other $d-1$ coordinates, by $\hat{e}_{i}$.

We can now define the consumer choice problem as follows. We consider a single consumer and $d$ different goods. A consumption bundle is a vector $x \in \mathcal{X}$, where $\mathcal{X}$ is the consumption space. We consider two settings for the consumption space: (i) infinitely divisible goods and (ii) indivisible goods. In the case of infinitely divisible goods, each of the goods is available in perfectly divisible quantities, and so $\mathcal{X}=\mathbb{R}_{+}^{d}$. In the case of indivisible goods, the goods can not be divided, and thus $\mathcal{X}=\mathbb{Z}_{+}^{d}$. In either case, we denote by $\leq$ the natural partial order on $\mathcal{X}$ : so $x \leq y$ iff $x_{i} \leq y_{i}$ for each coordinate $i \in[d]$. The $d$ goods generate a lattice $(\mathcal{X}, \leq)$.

An instance of the consumer choice problem is specified by a price vector $p \in \mathbb{R}_{++}^{d}$ and a consumer income $b \in \mathbb{R}_{+}$. Let $B(p, b):=\{x: x \in \mathcal{X}, p \cdot x \leq b\}$ denote the budget set under prices $p$ and budget $b$.

The standard economic theory of the consumer then postulates that the consumer behaves (i.e. chooses $x$ ) as if he solves the problem

$$
\max \{u(x): x \in B(p, b)\} .
$$

To incorporate computational considerations, we make the distinction between tractable and intractable utility functions, using the standard demarcation of polynomial-time computability. 
Definition 1 (Tractable Utility Functions). A utility function $u: \mathcal{X} \rightarrow \mathbb{R}$ is tractable if and only if problem (1) can be solved in polynomial time ${ }^{3}$ for all $p \in \mathbb{R}_{++}^{d}$ and $b \in \mathbb{R}_{+}$. Otherwise, $u$ is said to be intractable.

\subsection{Infinitely divisible goods}

We first discuss the case of infinitely divisible goods, which serves as a simple motivational example for the idea of a "revealed preference" view of computational complexity.

In the case of divisible goods, the consumption space is $\mathcal{X}=\mathbb{R}_{+}^{d}$. Thus, the standard economic theory of the consumer postulates that the consumer behaves (i.e. chooses $x$ ) as if solving the problem

$$
\max \left\{u(x): x \in \mathbb{R}_{+}^{d} \text { and } p \cdot x \leq b\right\} .
$$

We examine this model according to a worst-case view of computational complexity and a revealed preference view of computational complexity.

\section{A worst-case view}

From a worst-case perspective, it is immediately clear that if the utility function is allowed to be general, the consumer could be required to solve an arbitrary non-convex optimization, which is computationally intractable. (For completeness, we prove this rather basic fact for monotone utilities in Lemma 16 in Appendix B. A similar result is in [17].) Thus, without additional assumptions on the allowable utility functions, a worst-case view of computational complexity leads to a critique of the model as unrealistically assuming that consumers are solving a problem that is not computationally tractable. Taking a revealed preference view of computational complexity will lead to a different conclusion.

\section{A revealed preference view}

At its core, our revealed preference view is an empirical approach to computational complexity, thus it starts from data about observed behavior. Specifically, suppose that we have finitely many observations of the purchases made by a consumer at prices $p^{i}$ and incomes $b^{i}, i=0, \ldots, n$. That is, we have data of the form $\left\{\left(x^{i}, p^{i}, b^{i}\right)\right\}_{i=0}^{n}$.

We say that the data is rationalizable if there is a monotone increasing function $u(\cdot)$ such that $x^{i}$ is the solution to (2) at prices $p^{i}$ and income $b^{i}$. Then, revealed preference theory

\footnotetext{
${ }^{3}$ For continuous optimization problems, we take the notion of "solving in polynomial time" to mean obtaining an additive $\epsilon$-approximation in time polynomial in the natural problem parameters and $\log (1 / \epsilon)$. To be completely formal, we may also specify a precise model of computation, e.g., a RAM with word size $\Theta(\log n)$. However we feel that this level of formalism adds little in precision to our results and detracts from the main discussion, and so we avoid it in the remainder of the paper.
} 
provides the following well-known result [1, 32], which says that the data results either from a consumer that is manifestly irrational or from a consumer whose behavior can be explained using a concave utility function:

Theorem 2. (Afriat's Theorem) In the consumer choice problem with infinitely divisible goods, the data $\left\{\left(x^{i}, p^{i}, b^{i}\right)\right\}_{i=0}^{n}$ is rationalizable if and only if it is rationalizable by a monotone increasing and concave utility function.

Though Afriat's Theorem does not explicitly address computational constraints, there exist computationally efficient approaches to solving continuous monotone concave maximization problems with convex feasible sets [4]. Thus, an immediate consequence of Afriat's Theorem is that a data set $\left\{\left(x^{i}, p^{i}, b^{i}\right)\right\}_{i=0}^{n}$ is either manifestly irrational or can be explained using a utility function for which it is computationally efficient to solve (2). So, in the case where goods are infinitely divisible, the constraint that consumers can solve (2) in a computationally efficient manner has no empirical consequences, since it does not impose any additional restriction on the applicability of the model beyond the restrictions imposed by the assumption of rationality.

\section{Relating the worst-case and revealed preference views}

The simple motivational example of infinitely divisible goods provides an illustration of the contrast between the worst-case and revealed preference views. This example highlights that the two views can lead to fundamentally different conclusions about the role of computation. However, observe that if an economic model is polynomial time computable in a worst-case sense, then it is immediate to see that computational restrictions will have no empirical consequences in the revealed preference view. So, the revealed preference view is weaker than the worst-case view.

The revealed preference view is weaker because the power of an adversary to choose a hard instance is limited. Instead of being able to specify a hard instance precisely (e.g., specify a hard utility function for the consumer), the instance must be specified via a data set. Since this interface is not precise, even if hard instances exist, they may not have empirical consequences in the revealed preference view if a data set specified by a hard instance can always be explained by an easy instance (e.g., all data sets are rationalizable via a monotone, concave utility function).

This discussion highlights that the revealed preference view is similar in philosophy to smoothed analysis $[30,31]$. In smoothed analysis the adversary also cannot precisely specify a worst-case instance. Instead, the efficiency of a small, random perturbation of the instance specified by the adversary is considered.

\subsection{Indivisible goods}

We now move to the computationally more interesting case of indivisible goods, i.e., $\mathcal{X}=\mathbb{Z}_{+}^{d}$. It is a realistic case, as many consumption goods are clearly indivisible. The standard economic 
theory of the consumer postulates that the consumer behaves (i.e., chooses $x$ ) as if solving the problem

$$
\max \left\{u(x): x \in \mathbb{Z}_{+}^{d} \text { and } p \cdot x \leq b\right\}
$$

The analysis in this setting is significantly more involved than that for infinitely divisible goods. The main result, however, will be the same: that the consumer problem is computationally hard in a worst-case sense, but that computational constraints have no empirical consequences in the revealed preference view.

\section{A worst-case view}

As in the case of infinitely divisible goods, it is immediately clear that if the utility function is allowed to be general, the consumer could be required to solve an arbitrary discrete optimization, which is computationally intractable. In fact, optimization over a discrete space is intractable for seemingly simple utility functions: we show in Lemma 16 that solving (3) is NP-hard, even with linear utility functions. Thus, without additional assumptions on the allowable utility functions, a worst-case view of computational complexity leads to a critique of the model as unrealistically assuming that consumers are solving a problem that is not computationally tractable.

\section{A revealed preference view}

We now switch to a revealed preference view of computational complexity. As in the case of infinitely divisible goods, it provides a very different perspective than the worst-case view.

To define the revealed preference view, we must first define the form of the observed data about consumer behavior. We model the data (input) as a sequence of some number $n$ of consumer choices $\left\{\left(x^{i}, p^{i}, b^{i}\right)\right\}_{i=0}^{n}$ where $x^{i} \in \mathbb{Z}_{+}^{d}$ is the consumer choice observed under prices $p^{i}$ and budget $b^{i}$ (i.e., under budget set $B^{i}:=\left\{x: p^{i} \cdot x \leq b^{i}\right\}$ ) for each $i \geq 1$. We assume that if $x^{i}<y$ then $y \notin B^{i}$; so $x^{i}$ is maximal in $B^{i}$. For technical reasons, we add a fictitious observation $\left(x^{0}, p^{0}, b^{0}\right)$ with $x^{0}=\mathbf{0}, p^{0}=\mathbf{1}, b^{0}=0$; that is, a consumer with no money chooses the only feasible choice, namely nothing at all.

Adopting the language of revealed preference theory, we let $X=\left\{x^{0}, x^{1}, \ldots, x^{n}\right\}$ be the set of bundles purchased at any time in the input and observe that the data induces a revealed preference relation

$$
R_{0}:=\left\{\left(x^{i}, x^{j}\right):\left(x^{i}, x^{j}\right) \in X^{2}, x^{i} \in B^{j}\right\} .
$$

As in the case of infinitely divisible goods, we say that the data $\left\{\left(x^{i}, p^{i}, b^{i}\right)\right\}_{i=0}^{n}$ is rationalizable if there is a monotone increasing function $u(\cdot)$ such that $x^{i}$ is the solution to $\max \left\{u(x): x \in B\left(p^{i}, b^{i}\right)\right\}$, for $i=0, \ldots, n$.

Now, as in the case of indivisible goods, revealed preference theory provides a characterization of the set of data that is rationalizable. Specifically, the following well-known result (see, for example, [24] or [25]) highlights the relationship between rationalizability and the preference relation $R_{0}$ : 
Theorem 3. In the consumer choice problem with indivisible goods, data $\left\{\left(x^{i}, p^{i}, b^{i}\right)\right\}_{i=0}^{n}$ is rationalizable if and only if the binary relation $R_{0}$ is acyclic.

There are results analogous to Theorem 2 for infinitely divisible goods. The following result is due to [7] (and extended by [28]). Of course, concave utility makes no sense in the indivisible case, but supermodularity and submodularity are useful properties of utility one can potentially exploit.

Theorem 4. In the consumer choice problem with indivisible goods, the following statements are equivalent:

- The data $\left\{\left(x^{i}, p^{i}, b^{i}\right)\right\}_{i=0}^{n}$ is rationalizable.

- The data is rationalizable by a supermodular utility function.

- The data is rationalizable by a submodular utility function.

At this point, however, the parallel with the case of infinitely divisible good ends. Unlike in the case of infinitely divisible goods, we can not immediately apply Theorem 4 to arrive at utility functions for which it is computationally efficient for consumers to solve (3). The reason is that solving (3) for either submodular or supermodular utility functions is not computationally efficient. Specifically, submodular maximization subject to a budget constraint is NP-hard, even to approximate to better than a $(1-1 / e)$ factor [15]. Further, supermodular maximization subject to a budget constraint is likewise NP-hard [20], and extremely inapproximable under reasonable complexity assumptions, as we show in Theorem 17.

The main result of this paper is that, when data is rationalizable, it is possible to rationalize the data using a utility function for which solving (3) is computationally efficient. Specifically: Theorem 5. In the consumer choice problem with indivisible goods, data $\left\{\left(x^{i}, p^{i}, b^{i}\right)\right\}_{i=0}^{n}$ is rationalizable if and only if it is rationalizable via a tractable monotone utility function.

The message in Theorem 5 is that computational constraints do not impose any further restrictions on the ability of consumer choice theory to explain data than are already imposed by the assumption of rationality. This is in stark contrast to the message suggested by the worst-case view of computational complexity for this model.

In order to prove Theorem 5, we must prove that the data is either not rationalizable, or there is a utility function rationalizing the data such that for any $p$ and $b$, (3) can be solved in strongly polynomial time. That is, using only computation time polynomial in $n, d$, and the number of bits required to write down the prices and budgets.

We cannot use Theorem 4 to prove Theorem 5. Instead, we proceed by working with the demand function instead of the utility function. Specifically, to prove the result we construct an efficient algorithm $\mathcal{A}=\mathcal{A}_{\left\{\left(x^{i}, p^{i}, b^{i}\right)\right\}_{i=0}^{n}}$ to compute a demand function $\psi: \mathbb{R}_{++}^{d} \times \mathbb{R}_{+} \rightarrow \mathbb{Z}_{+}^{d}$ such that

\footnotetext{
${ }^{4}$ That is to say, $\psi(p, b)$ is the choice of the consumer faced with budget set $B(p, b)$.
} 
(i) the demand function explains the data, i.e., $\psi\left(p^{i}, b^{i}\right)=x^{i}$ for all $i \in[n]$, and

(ii) there is a monotone increasing utility function $u(\cdot)$ rationalizing the data, and for all $p \in \mathbb{R}_{++}^{d}$ and $b \in \mathbb{R}_{+}$we have

$$
\psi(p, b)=\arg \max \{u(x): x \in B(p, b)\} .^{5}
$$

We prove this fact as Corollary 15 in Appendix A. Interestingly, the algorithm does not explicitly construct a tractable utility function; rather, a utility function is implicit in the demand function which our algorithm computes.

In the remainder of this section, we explain the algorithm for computing a demand function from the data. As described in the pseudocode, the algorithm consists of two steps: (i) A preprocessing phase, $\mathcal{A}$.preprocess, in which a ranking $r$ on $X=\left\{x^{0}, x^{1}, \ldots, x^{n}\right\}$ is computed. (ii) A core phase, $\mathcal{A}$.evaluate_demand, that uses the ranking in order to evaluate the demand function $\psi(p, b)$. Define lex max $S$ denote the lexicographically maximum element of $S$.

A first remark on Algorithm $\mathcal{A}$ is that, as a side effect, algorithm $\mathcal{A}$ is, in some sense, "learning" the consumer demand function. Indeed, as more data is observed the choice function determined by $\mathcal{A}$ is increasingly accurate. But, if one looks at the design of $\mathcal{A}$ from this perspective, the use of lex max in $\mathcal{A}$.evaluate_demand is artificial. Interestingly, the proof of Theorem 5 does not depend on properties of lex max, and instead other functions that projects $z(p, b)$ onto the boundary of $B(p, b)$ can be used instead. Thus, one could parameterize the algorithm with prior beliefs about consumer demand.

We defer the proof of the correctness of Algorithm $\mathcal{A}$ to Appendix $\mathrm{A}$ and discuss only the running time of the algorithm here. It is immediate to see that phase (ii), $\mathcal{A}$.evaluate_demand, can be implemented in $\mathcal{O}(n d)$ time. Phase (i), $\mathcal{A}$.preprocess, can be implemented in $\mathcal{O}\left(n^{2} d\right)$ time, since we may iterate over $X^{2}$, and test if each element belongs to $E_{0} \cup R_{0}$ in $\mathcal{O}(d)$ time, and topological sort is well-known to have a linear-time implementation, i.e., one taking $\mathcal{O}(|X|+|E|)$ time, which is this case amounts to $\mathcal{O}\left(n^{2}\right)$. The total time is thus $\mathcal{O}\left(n^{2} d\right)$.

One, possibly surprising, observation about the running time of algorithm $\mathcal{A}$ is that it depends on $n$, the size of the data set. One may reasonably take $n$ to be a constant when evaluating the running time of the demand function $\psi$ since it is the tractability with respect to $p$ and $b$ that is relevant when the choice function is evaluated for future demands (i.e., the data set is fixed for all future evaluations of the demand function). However, one may also reasonably ask whether the dependence of the running time on $n$ is necessary or if it is an artifact of specific algorithm presented here.

It turns out that there is an unavoidable dependence of any rationalization algorithm on the size of the data in (at least) two ways. First, when determining a demand function to rationalize the data, the algorithm must process every 'distinct' element of the data set, which highlights

\footnotetext{
${ }^{5}$ Note the divergence from the more classical approach in revealed preference theory; e.g [1, 7]. Most studies of revealed preference construct a rationalizing utility. Here the approach is to construct a demand function, and then show that it is rationalizable.
} 


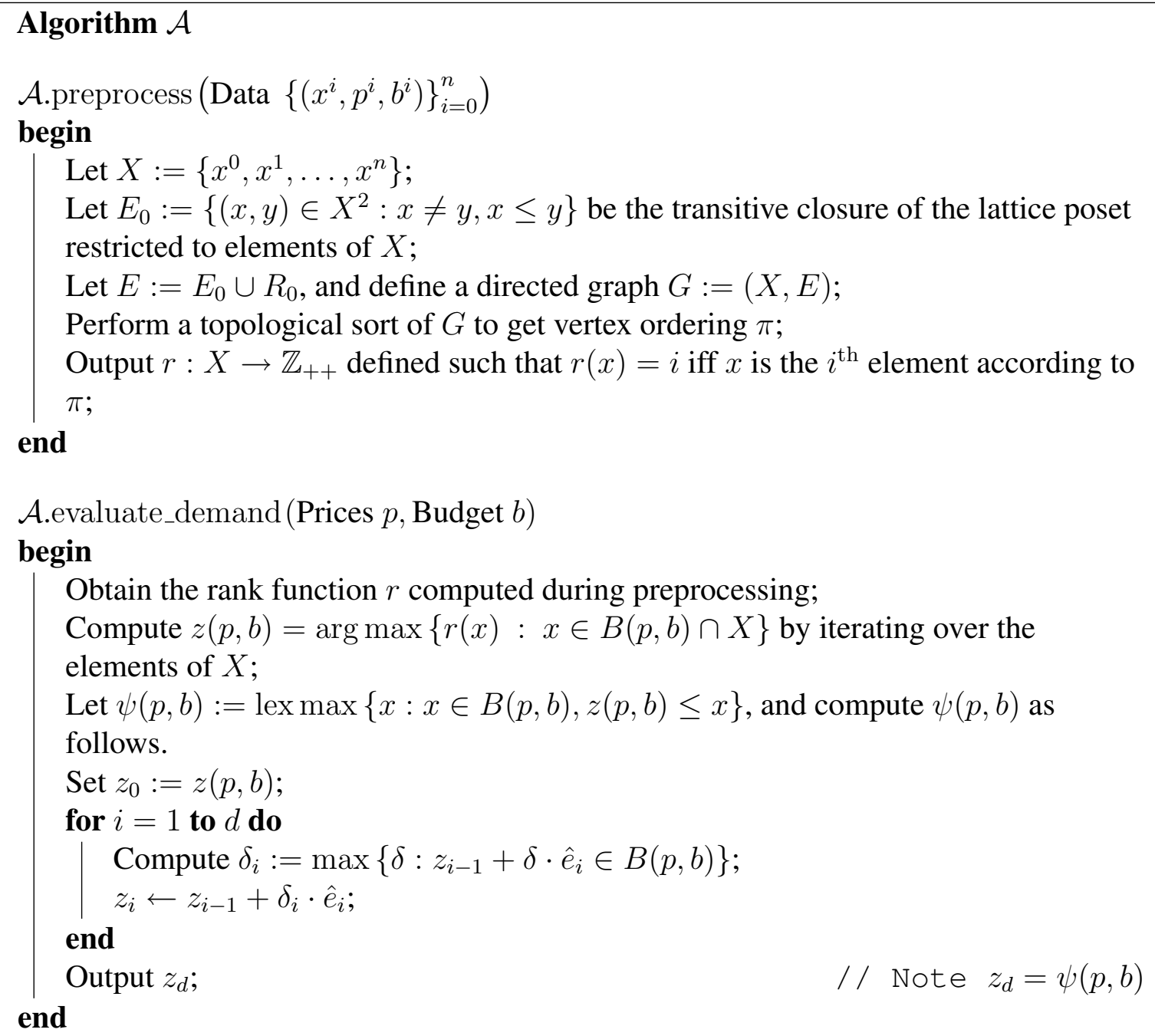


that the running time must be at least the size of the minimal representation of the data set. Second, the resulting demand function must at least encode each entry of the data set. Thus, the space needed to represent the demand function must also be bounded below by the size of the minimal representation of the data set. More formally, we can prove the following lower bounds on the running time of any algorithm for rationalizing the data set and for the space needed to represent the resulting demand function. The proofs are deferred to Appendix C.

Proposition 6. Any algorithm that takes as input a data set with $n$ data points, a price vector $p$, and $a$ budget $b$ and outputs $\psi(p, b)$ for a $\psi$ which rationalizes the data set requires, in the worst case, $\Omega(n)$ running time on a RAM with word size $\Theta(\log n)$, even when there are only two goods.

Proposition 7. Any demand function $\psi$ that rationalizes a data set with $n$ data points requires $\Omega(n \log n)$ bits of space to represent, in the worst case, even when there are only two goods.

These two propositions highlight that the dependence of the running time on the size of the data set is unavoidable. However, observe that the running time of algorithm $\mathcal{A}$ does not match the lower bound above, since it has a quadratic dependence on $n$. On the other hand, our preprocessing step is space-optimal, since it can be implemented using linear space by performing the topological sort in a lazy manner that generates the adjacently list of each vertex of $G$ on the fly, and hence avoids the need to explicitly construct $G$. The running time of this lazy implementation is also $\mathcal{O}\left(n^{2} d\right)$.

\section{Walrasian equilibrium theory}

The theory of the consumer is an important component in many economic theories. The most important of these is general equilibrium theory, which in turn is the most widely applied model in mainstream micro economics (it underlines most of modern macro economics, international trade and financial economics). In this section, we present the implications of our results on consumer choice theory for general equilibrium theory, specifically the implications for the empirical content of Walrasian equilibrium as formulated by [5].

The basic model of general equilibrium assumes a finite number of goods and agents. Agents have preferences and are endowed with initial quantities of the goods. (We ignore production in our discussion.) The theory predicts which prices will emerge for the different goods, and what the quantities of the goods consumed by the agents will be. Specifically, the theory predicts that the prices and quantities conform to a Walrasian equilibrium: a situation where agents choose quantities optimally given prices, endowments, and the incomes derived from selling their endowments; and where supply equals demand for all goods simultaneously.

We can describe the revealed preference formulation of Walrasian equilibrium as follows. Given data on prices and resources for an economy, we want to know if the data are rationalizable as Walrasian equilibrium outcomes. The rationalization involves specifying utility functions for the agents. We want to study if these utility functions can be chosen so that they are tractable. 
Consider the standard model of an exchange economy. Suppose there are $d$ goods and $m$ agents. Each agent $i$ is described by a consumption space $\mathcal{X}_{i} \subseteq \mathbb{R}_{+}^{d}$, a monotone increasing utility function $u_{i}: \mathcal{X}_{i} \rightarrow \mathbb{R}$, and an endowment $\omega_{i} \in \mathcal{X}_{i}$. An economy is therefore described by $\left(\mathcal{X}_{i}, u_{i}, \omega_{i}\right)_{i=1}^{m}$. By changing the consumption space $\mathcal{X}_{i}$, we can work with either indivisible or divisible goods. To apply the results in Section 2 we assume that $\mathcal{X}_{i}=\mathbb{Z}_{+}^{d}$.

An allocation is a collection $\mathbf{x}=\left(x_{i}\right)_{i=1}^{m}$ with $x_{i} \in \mathcal{X}_{i}$, and such that $\sum_{i=1}^{m} x_{i}=\sum_{i=1}^{m} \omega_{i}$. A Walrasian equilibrium is a pair $(\mathbf{x}, p)$, where $\mathbf{x}=\left(x_{i}\right)_{i=1}^{m}$ is an allocation and $p \in \mathbb{R}_{++}^{d}$ is a price vector such that, for all $i, x_{i}$ solves the problem

$$
\max \left\{u_{i}(x): x \in \mathcal{X}_{i}, p \cdot x \leq p \cdot \omega_{i}\right\}
$$

We assume that we have data on prices, incomes and resources. Specifically, a data set is a collection $\left(p^{k},\left(\omega_{i}^{k}\right)_{i=1}^{m}\right)_{k=0}^{K}$. We follow [5] in assuming that individual consumption vectors are not observable. We say that a data set is rationalizable if there are utility functions $u_{i}$ for each agent, and vectors $x^{k}, k=1, \ldots, K$, such that $\left(x^{k}, p^{k}\right)$ is a Walrasian equilibrium of the economy $\left(\mathcal{X}_{i}, u_{i}, \omega_{i}^{k}\right)_{i=1}^{m} \cdot{ }^{6}$

Given the above setup, the results in Section 2 imply that in the case of indivisible goods (i.e., when $\left.\mathcal{X}_{i}=\mathbb{Z}_{+}^{d}\right)$, a data set $\left(p^{k},\left(\omega_{i}^{k}\right)_{i=1}^{m}\right)_{k=0}^{K}$ is either not rationalizable, or it is rationalizable using tractable $u_{i}$ for all agents: utilities such that the problems

$$
\max \left\{u_{i}(x): x \in \mathbb{Z}_{+}^{d}, p \cdot x \leq p \cdot \omega_{i}\right\}
$$

can be solved in strongly polynomial time. Further, from the discussion in Section 2 it is easy to see that a similar result holds for infinitely divisible goods (i.e., $\mathcal{X}_{i}=\mathbb{R}_{+}^{d}$ ) as a consequence of Afriat's Theorem.

However, it is important to realize that our results do not address the complexity of finding a Walrasian equilibria. We have limited the discussion to the individual consumer's problem, not the collective problem of finding an equilibrium. The later question is clearly important and interesting, and it is a focus of continuing work on this topic. The exact formulation of the revealed preference view for this extension is a special case of the general formulation that we introduce in Section 4.

\section{A general formulation}

In this paper we focus on one particular theory, consumer choice theory; however it is clear that the revealed preference view of computational complexity can be studied much more broadly. In this section we present a general formulation of the revealed preference view which can be applied, for example, to test the impact of computational constraints on the empirical content of the theory of Nash equilibrium, the theory of stable matchings, and other economic theories.

\footnotetext{
${ }^{6}$ One can, instead, assume that only individual incomes are observable, and not individual endowments. The results are the same (see [5]).
} 
To begin the general formulation, let $\mathcal{I}$ be a set of possible inputs, and $\mathcal{O}$ be a set of possible outputs. For example, in consumer theory, $\mathcal{I}$ is the set of all price-budget pairs $(p, b)$, while $\mathcal{O}$ is the consumption space $(\mathcal{X})$.

We represent a theory as a collection $\mathcal{G}=\left\{g_{\tau}: \tau \in \mathcal{T}\right\}$ of correspondences, $g_{\tau} \subseteq \mathcal{I} \times \mathcal{O}$, which we index by a set $\mathcal{T}$ for convenience. For example, consumer choice theory is the collection $\left\{\psi_{u}: u \in U\right\}$ where $U$ is the set of all monotone increasing utility functions $u: \mathcal{X} \rightarrow \mathbb{R}$ and $\psi_{u}(p, b)$ is the demand function obtained from maximizing $u$ over the budget set defined by $(p, b)$.

\section{Classical revealed preference theory}

When taking a revealed preference perspective on a theory, the goal is to test the theory using data. We define a data set $D$ as a finite collection $D=\left(i_{k}, o_{k}\right)_{k=1}^{n}$ of pairs of inputs and outputs. The set of all possible data sets is $\mathcal{D}=(\mathcal{I} \times \mathcal{O})^{*}$, i.e., all finite sequences of pairs in $\mathcal{I} \times \mathcal{O}$.

A data set $\left(i_{k}, o_{k}\right)_{k=1}^{n}$ is rationalizable by the theory $\mathcal{G}=\left\{g_{\tau}: \tau \in \mathcal{T}\right\}$ if there is $\tau \in \mathcal{T}$ such that $o_{k} \in g_{\tau}\left(i_{k}\right)$ for all $k=1, \ldots, n$. In that case, we say that $g_{\tau}$ rationalizes the data.

Further, we define a rationalization rule $r: \mathcal{D} \rightarrow(\mathcal{I} \rightarrow \mathcal{O})$ such that for each rationalizable data set $D, r(D)=g_{\tau}$ for some $\tau \in \mathcal{T}$ such that $g_{\tau}$ rationalizes the data.

Finally, define the set of data sets rationalizable by theory $\mathcal{G}$ as

$$
\mathcal{R}_{\mathcal{G}}=\left\{D \in \mathcal{D}: \exists \tau \in \mathcal{T}: g_{\tau} \text { rationalizes } D\right\}
$$

Given this setup, some classic revealed preference questions are:

Is theory $\mathcal{G}$ refutable, i.e., Is $\mathcal{R}_{\mathcal{G}} \varsubsetneqq \mathcal{D}$ ?

Is data $D$ rationalizable by theory $\mathcal{G}$, i.e., Is $D \in \mathcal{R}_{\mathcal{G}}$ ?

However, these classic questions ignore computational constraints on the theory.

\section{Adding computational constraints}

Our revealed preference view of computational complexity can be fit into the above framework as follows.

Consider again the rationalization rule $r: \mathcal{D} \rightarrow(\mathcal{I} \rightarrow \mathcal{O})$. We now seek to understand the impact of imposing an additional constraint on $r(D)=g_{\tau}$ which rationalizes $D$ - it must be "tractable." Formally, we say that $g_{\tau}(i)=[r(D)](i)$ is tractable if it is computable in time polynomial in the size of both $D$ and $i$. We require it to be polynomial in the size of $D$ for reasons discussed below (and in Section 2). 
Define the set of data sets that are rationalizable using tractable instances of theory $\mathcal{G}$ as

$$
\mathcal{E}_{\mathcal{G}}=\left\{D \in \mathcal{D}: \exists \tau \in \mathcal{T} . g_{\tau} \text { rationalizes } D \text { and } g_{\tau} \text { is tractable }\right\}
$$

Given this framework, the general version of the question posed by the revealed preference view of complexity is:

Do computational constraints have empirical consequences, i.e., Is $\mathcal{E}_{\mathcal{G}} \varsubsetneqq \mathcal{R}_{\mathcal{G}}$ ?

In general, the set $\mathcal{E}_{\mathcal{G}}$ constitutes a test for the joint hypotheses of assuming the economic theory $\mathcal{G}$, and requiring that the economic system not solve unrealistically hard problems. If $\mathcal{E}_{\mathcal{G}} \varsubsetneqq \mathcal{R}_{\mathcal{G}}$ then one would seek to characterize $\mathcal{E}_{\mathcal{G}}$ so that actual tests of the joint hypotheses can be carried out.

One final remark about this formulation is that it may initially seem strange that the computational time of $g_{\tau}$ depends on size of $D$, but, as we saw in the case of consumer choice theory, this is necessary in order to ensure that $g_{\tau}$ rationalizes $D$.

In fact, we can generalize Propositions 6 and 7 for this general formulation as follows. For a theory $\mathcal{G}$, define

$$
\mathcal{R}_{\mathcal{G}}\left(\left\{i_{k}\right\}_{k=1}^{n}\right):=\mathcal{R}_{\mathcal{G}} \cap\left\{\left\{\left(i_{k}, o_{k}\right)\right\}_{k=1}^{n}: \forall k, o_{k} \in \mathcal{O}\right\}
$$

to be the set of rationalizable data sets on input sequence $\left\{i_{k}\right\}_{k=1}^{n}$. Then we have the following lower bounds on the running time of $r$ and space required to represent $g_{\tau} \in \mathcal{G}$. The proofs are deferred to Appendix C.

Proposition 8. Fix a theory $\mathcal{G}$ and $n \in \mathbb{N}$. The running time required to compute $[r(D)](i)$ for Dof size $n$ is

$$
\Omega\left(\frac{\max _{\left(i_{1}, \ldots, i_{n}\right)} \log _{2}\left|\mathcal{R}_{\mathcal{G}}\left(\left\{i_{k}\right\}_{k=1}^{n}\right)\right|}{\log n}\right)
$$

in the worst case on a RAM with wordsize $\Theta(\log n)$, where the max is taken over all input sequences $\left\{i_{k}\right\}_{k=1}^{n}$ of length $n$.

Proposition 9. Fix a theory $\mathcal{G}$ and $n \in \mathbb{N}$. The space required to represent any $g_{\tau} \in \mathcal{G}$ rationalizing a rationalizable data set of size $n$ is at least $\max _{\left(i_{1}, \ldots, i_{n}\right)} \log _{2}\left|\mathcal{R}_{\mathcal{G}}\left(\left\{i_{k}\right\}_{k=1}^{n}\right)\right|$ bits in the worst case, where the max is taken over all input sequences $\left\{i_{k}\right\}_{k=1}^{n}$ of length $n$.

These propositions highlight that there is an explicit tradeoff between the generality of the theory being tested and the running time of $r$ and the space complexity of $g_{\tau}$. The generality of the theory is measured by the quantity $\max _{\left(i_{1}, \ldots, i_{n}\right)}\left|\mathcal{R}_{\mathcal{G}}\left(\left\{i_{k}\right\}_{k=1}^{n}\right)\right|$, which captures the notation that a more general theory can explain (rationalize) a larger class of data sets. 


\section{Concluding remarks}

The core idea presented by this paper is that there is a philosophical difference in how many computer scientists and economists view economic models, and that the lack of broad acceptance of worst-case complexity results in economics stems from the difference between these viewpoints. We posit that while computer scientists tend to think 'algorithmically' about economic models, economists tend to think 'empirically' about the models. ${ }^{7}$

In particular, an algorithmic view of the model assumes the model is fixed and literally true, and then proceeds to ask about the demands placed on the agents by the model. That is, it assumes that the agent is simply an implementation of the model and asks whether the agent can efficiently compute its decisions.

In contrast, an empirical view of the model takes the model as a tool for thinking about reality. One does not presume agents literally follow the model, only that the model provides a way to explain the observed behavior, i.e., the data. Thus, an empirical view of economic models is that they provide 'as-if' tools: economists postulate a model and claim the observable variables behave as if the model were true.

Note that the empirical view does not deny that computational issues should be studied. To the contrary, a model still loses credibility if the agents must solve computationally hard problems; however, worst-case complexity is no longer the relevant concept. Instead, the question is whether data from an observed phenomenon can always be explained by the theory with the additional constraint that agents are not required to solve computationally hard problems.

This paper presents a formulation of how to incorporate computational information into the empirical view; however the algorithmic view continues to be very relevant, of course. Many problems require the design of automated agents who participate in various economic interactions: in this case the utilities are hard-wired by the designer, they are not theoretical unknowns. Using classical economic jargon, one could say that the algorithmic view is relevant for normative economics while the empirical view is relevant for positive economics.

\section{References}

[1] S. N. Afriat. The construction of utility functions from expenditure data. International Economic Review, 8(1):67-77, feb 1967.

[2] S. N. Afriat. Efficiency estimation of production functions. International Economic Review, 13(3):568-598, oct 1972.

[3] M.-F. Balcan and A. Blum. Approximation algorithms and online mechanisms for item pricing. Theory of Computing, 3(9):179-195, 2007.

\footnotetext{
${ }^{7}$ There are clearly additional factors that also contribute to the lack of acceptance of computational constraints by economists. For example, some economists are not familiar with basic ideas, such as the implications of Church's thesis for why complexity matters for economic systems.
} 
[4] S. Boyd and L. Vandenberghe. Convex Optimization. Cambridge University Press, 2004.

[5] D. J. Brown and R. L. Matzkin. Testable restrictions on the equilibrium manifold. Econometrica, 64(6):1249-1262, November 1996.

[6] D. J. Brown and C. Shannon. Uniqueness, stability, and comparative statics in rationalizable Walrasian markets. Econometrica, 68(6):1529-1439, November 2000.

[7] C. P. Chambers and F. Echenique. Supermodularity and preferences. Journal of Economic Theory, 144(3):1004-1014, May 2009.

[8] C. P. Chambers, F. Echenique, and E. Shmaya. On behavioral complementarity and its implications. Forthcoming, Journal of Economic Theory, 2009.

[9] X. Chen, D. Dai, Y. Du, and S.-H. Teng. Settling the complexity of arrow-debreu equilibria in markets with additively separable utilities. In FOCS '09: Proceedings of the 2009 50th Annual IEEE Symposium on Foundations of Computer Science, pages 273-282, Washington, DC, USA, 2009. IEEE Computer Society.

[10] X. Chen, X. Deng, and S.-H. Teng. Settling the complexity of computing two-player Nash equilibria. J. $A C M, 56(3): 1-57,2009$.

[11] C. Daskalakis. Nash equilibria: Complexity, symmetries, and approximation. Computer Science Review, 3(2):87-100, 2009.

[12] C. Daskalakis, P. W. Goldberg, and C. H. Papadimitriou. The complexity of computing a Nash equilibrium. SIAM J. Comput., 39(1):195-259, 2009.

[13] W. E. Diewert and C. Parkan. Linear progamming tests of regularity conditions for production functions. In W. Eichhorn, R. Henn, K. Neumann, and R. Shephard, editors, Quantitative Studies on Production and Prices, pages 131-158. Physica-Verlag, Vienna, 1983.

[14] K. Etessami and M. Yannakakis. On the complexity of Nash equilibria and other fixed points. SIAM J. Comput., 39(6):2531-2597, 2010.

[15] U. Feige. A threshold of $\ln n$ for approximating set cover. Journal of the ACM, 45(4):634 -652 , July 1998.

[16] M. R. Garey and D. S. Johnson. Computers and Intractability: A Guide to the Theory of NP-Completeness. W. H. Freeman \& Co., New York, NY, USA, 1979.

[17] I. Gilboa, A. Postlewaite, and D. Schmeidler. The complexity of the consumer problem and mental accounting. Mimeo: Tel-Aviv University, 2010.

[18] V. Guruswami, J. D. Hartline, A. R. Karlin, D. Kempe, C. Kenyon, and F. McSherry. On profit-maximizing envy-free pricing. In SODA '05: Proceedings of the sixteenth annual ACM-SIAM symposium on Discrete algorithms, pages 1164-1173, Philadelphia, PA, USA, 2005. Society for Industrial and Applied Mathematics. 
[19] K. Jain. A polynomial time algorithm for computing an Arrow-Debreu market equilibrium for linear utilities. SIAM J. Comput., 37(1):303-318, 2007.

[20] H. Kellerer, U. Pferschy, and D. Pisinger. Knapsack problems, chapter 12. Springer, 1 edition, 2004.

[21] S. Khot. Ruling out PTAS for graph min-bisection, dense $k$-subgraph, and bipartite clique. SIAM J. Comput., 36(4):1025-1071, 2006.

[22] R. L. Matzkin. Axioms of revealed preference for nonlinear choice sets. Econometrica, 59(6):1779-1786, November 1991.

[23] N. Nisan, T. Roughgarden, E. Tardos, and V. V. Vazirani, editors. Algorithmic Game Theory. Cambridge University Press, New York, NY, USA, 2007.

[24] M. K. Richter. Revealed preference theory. Econometrica, 34(3):635-645, jul 1966.

[25] M. K. Richter. Rational choice. In J. S. Chipman, L. Hurwicz, M. K. Richter, and H. F. Sonnenschein, editors, Preferences, Utility and Demand, pages 29-58. Harcourt Brace Jovanivic Inc., 1971.

[26] T. Roughgarden. Computing equilibria: a computational complexity perspective. Economic Theory, 42(1):193-236, January 2010.

[27] P. Samuelson. A note on the pure theory of consumer's behaviour. Economica, 5(17):61$71,1938$.

[28] K. Shirai. On the existence of a submodular utility function. Mimeo: Waseda University, 2010 .

[29] H. A. Simon. Rationality in psychology and economics. The Journal of Business, 59:209224, 1986.

[30] D. A. Spielman and S.-H. Teng. Smoothed analysis of algorithms: Why the simplex algorithm usually takes polynomial time. J. ACM, 51(3):385-463, 2004.

[31] D. A. Spielman and S.-H. Teng. Smoothed analysis: an attempt to explain the behavior of algorithms in practice. Commun. ACM, 52(10):76-84, 2009.

[32] H. R. Varian. The nonparametric approach to demand analysis. Econometrica, 50(4):945974, jul 1982.

[33] H. R. Varian. Non-parametric tests of consumer behaviour. Review of Economic Studies, 50(1):99-110, jan 1983.

[34] H. R. Varian. Revealed preference. In M. Szenberg, L. Ramrattan, and A. Gottesman, editors, Samuelsonian economics and the twenty-first century, pages 99-115. Oxford University Press, USA, 2006. 
[35] V. V. Vazirani and M. Yannakakis. Market equilibrium under separable, piecewise-linear, concave utilities. In Proc. Innovations in Computer Science, pages 156-165, Beijing, China, January 2010.

[36] Y. Ye. A path to the Arrow-Debreu competitive market equilibrium. Mathematical Programming, 111(1):315-348, 2008. 


\section{Appendix A Proofs for the consumer choice model}

In this section we prove Theorem 5 via a sequence of lemmas culminating in Theorem 14 and Corollary 15.

Lemma 10. If $R_{0}$ is acyclic, then $\preceq:=\left(R_{0} \cup \leq\right)^{*}$ is acyclic.

Proof. The proof follows from the definition of budget set. Here, we use the convention that $x R_{0} y$ is the same as $(x, y) \in R_{0}$, meaning $x$ is revealed-preferred to $y$.

Now suppose by way of contradiction that $R_{0}$ is acyclic, but $\preceq$ contains a minimal cycle $C$. Since $\leq$ is transitive, any minimal cycle consists only of edges in $X^{2}$ (recall $X$ is the set of consumption bundles observed in the data plus the zero lattice point). Otherwise $C$ contains edges $(x, y)$ and $(y, z)$ for some $y \notin X$. Since these pairs cannot be in $R_{0}$, we have $x \leq y$ and $y \leq z$. Hence $x \leq z$ and we can replace $(x, y)$ and $(y, z)$ with $(x, z)$, which violates the minimality of $C$.

Since $R_{0}$ is acyclic by assumption, $C$ must contain an edge $(x, y) \notin R_{0}$. Because $(x, y) \in$ $\left(R_{0} \cup \leq\right)^{*}$ by assumption, this means $x \leq y$. However, since $x, y \in X$ from our earlier argument, and because the budget sets are downward-closed with respect to $\leq, x \leq y$ implies $(x, y) \in R_{0}$ by definition of $R_{0}$. This contradictions our earlier assumption that $(x, y) \notin R_{0}$. Hence no minimal cycle exists, and $\preceq$ is acyclic.

Lemma 11. Algorithm $\mathcal{A}$ computes a choice function $\psi$ such that $\psi(p, b) \in B(p, b)$ for all $p$ and $b$.

Proof. For all valid $p$ and $b, \mathbf{0} \in B(p, b) \cap X$, and all elements $x \in X$ have distinct ranks $r(x)$, so $z(p, b)$ and hence $x(p, b)$ are well defined, and by construction, the output $x(p, b) \in B(p, b)$.

Lemma 12. Let $r$ be the ranking computed during preproccessing. Let $\preceq:=\left(R_{0} \cup \leq\right)^{*}$ and assume $R_{0}$ is acyclic. Fix $x, y \in X$. Then ranks are distinct, i.e., $x \neq y$ implies $r(x) \neq r(y)$, and the ranks are monotone in $\preceq$, i.e., $x \preceq y$ implies $r(x) \leq r(y)$.

Proof. The first part, $x \neq y$ implies $r(x) \neq r(y)$, holds by construction since $r(\cdot)$ is the rank within a permutation $\pi$ and no two elements have the same rank. Now suppose $x \preceq y$. Then by definition of $\preceq$ there is a path $P$ in $\left(R_{0} \cup \leq\right)^{*}$ from $x$ to $y$. We claim there must be a path $P^{\prime}$ from $x$ to $y$ in the digraph $G=(X, E)$ constructed during preproccessing, since $(i)$ all the edges in $R_{0}$ are present in $E$, and (ii) every maximal subpath of $P$ starting and ending at lattice points in $X$ and consisting only of edges in $\leq$ can be traversed by a single edge in $E_{0}$ by the transitivity of $\leq$, and $E_{0} \subseteq E$, and (iii) $P$ can be decomposed into edges in $R_{0}$ and maximal subpaths of $P$ starting and ending at lattice points in $X$. Hence there is a path in $G$ from $x$ to $y$ and so the topological sort must place $x$ before $y$ in $\pi$, which implies $r(x) \leq r(y)$. (Note that since $R_{0}$ is acyclic by assumption, $\preceq$ is acyclic by Lemma 10 , which in turn implies $G$ is acyclic, and thus there exists a topological ordering of its vertices.) 
Lemma 13. Assume that $R_{0}$ is acyclic. The choice function $\psi$ computed by $\mathcal{A}$ explains the data, i.e., $\psi\left(p^{i}, b^{i}\right)=x^{i}$ for all $i \in[n]$.

Proof. Fix $i \in[n]$ arbitrarily. Recall $B^{i}$ is the budget set faced by the consumer when $x^{i}$ was chosen. Note $x^{i} \in B^{i} \cap X$, so it is considered in the arg max in the computation of $z\left(p^{i}, b^{i}\right)$. We consider two cases, namely $z\left(p^{i}, b^{i}\right)=x^{i}$ and $z\left(p^{i}, b^{i}\right) \neq x^{i}$.

In the first case, we have $\psi\left(p^{i}, b^{i}\right)=x^{i}$, as $x^{i}$ is a maximal element of the budget set with respect to the lattice ordering $\leq$, and hence the evaluation of the lex max in the computation of $x\left(p^{i}, b^{i}\right)$ is over the singleton set $\left\{x^{i}\right\}$, and so the output of $\mathcal{A}$ is $x\left(p^{i}, b^{i}\right)=x^{i}$.

We next argue that the second case cannot occur. Suppose by way of contradiction that $z\left(p^{i}, b^{i}\right) \neq x^{i}$. Then there must be some $x^{j} \neq x^{i}$ such that $r\left(x^{i}\right)<r\left(x^{j}\right)$ and $x^{j} \in B^{i}$, by the definition of $z\left(p^{i}, b^{i}\right)$. However, the fact that $x^{j} \in B^{i}$ and $x^{i}$ was chosen in the $i^{\text {th }}$ observation implies $\left(x^{j}, x^{i}\right) \in R_{0}$ and hence $x^{j} \preceq x^{i}$, where $\preceq:=\left(R_{0} \cup \leq\right)^{*}$ as before. Lemma 12 then implies $r\left(x^{j}\right) \leq r\left(x^{i}\right)$, contradicting our earlier deduction that $r\left(x^{i}\right)<r\left(x^{j}\right)$.

We introduce $R_{\psi}$, the preference relation revealed by $\psi(\cdot)$ :

$$
R_{\psi}:=\{(x, y): \exists p, b . x \in B(p, b) \backslash\{y\} \text { and } y=\psi(p, b)\} .
$$

Note $\psi\left(p^{i}, b^{i}\right)=x^{i}$ for all $i \in[n]$ implies $R_{0} \subset R_{\psi}$.

Theorem 14. Algorithm $\mathcal{A}$ computes a choice function $\psi$ such that if $R_{\psi}$ is the preference relation revealed by $\psi$ and $R_{0}$ is acyclic, then $\psi$ explains the data and

Proof. We prove $\psi$ explains the input in Lemma 13. Suppose, by way of contradiction, that $\preceq$ is acyclic and yet there is a minimal cycle $C$ in the induced binary relation $\left(R_{\psi} \cup \leq\right)^{*}$. Suppose that $C$ consists of $k$ edges $\left\{\left(y^{i}, y^{i+1}\right): 1 \leq i \leq k\right\}$, where we define $y^{k+1}:=y^{1}$ for convenience. We can suppose that each edge $\left(y^{i}, y^{i+1}\right)$ is in $R_{\psi}$; the proof of this fact is similar to the proof of Lemma 10. Let $p\left(y^{i}\right)$ and $b\left(y^{i}\right)$ denote the prices and budget corresponding to the choice of $y^{i}$, so that $\psi\left(p\left(y^{i}\right), b\left(y^{i}\right)\right)=y^{i}$. Let $B\left(y^{i}\right):=B\left(p\left(y^{i}\right), b\left(y^{i}\right)\right)$ denote the corresponding budget set. Let $z^{i}:=z\left(p\left(y^{i}\right), b\left(y^{i}\right)\right)$ be the intermediate lattice point computed when running $\mathcal{A}$ with input $\left(p\left(y^{i}\right), b\left(y^{i}\right)\right)$ and the ranking $r(\cdot)$. We consider two cases, namely $z^{i}=z^{j}$ for all $i, j \in[k]$, or there exists $i, j$ with $z^{i} \neq z^{j}$.

We start with the case that $z^{i}=z^{j}$ for all $i, j \in[k]$. By construction,

$$
y^{i} \equiv x\left(p\left(y^{i}\right), b\left(y^{i}\right)\right)=\operatorname{lex} \max \left\{x: x \in B\left(y^{i}\right), z^{i} \leq x\right\}
$$

for all $i$. Since $\left(y^{i}, y^{i+1}\right) \in C$, we know $y^{i} \in B\left(y^{i+1}\right)$, i.e., $y^{i}$ is affordable for the budget set for which the consumer purchases $y^{i+1}$. Since $z^{i}=z^{i+1}$, this implies $y^{i}$ is in the set over which the algorithm computes the lex max when running on input $\left(p\left(y^{i+1}\right), b\left(y^{i+1}\right)\right)$, namely $\left\{x: x \in B\left(y^{i+1}\right), z^{i+1} \leq x\right\}$. Since $y^{i+1}$ was selected, $y^{i}$ is lexicographically less than or equal to $y^{i+1}$, which we denote by $y^{i} \leq_{\text {Lex }} y^{i+1}$. Since $i$ was arbitrary,

$$
y^{1} \leq_{\text {Lex }} y^{2} \leq_{\text {Lex }} \cdots \leq_{\text {Lex }} y^{k} \leq_{\text {Lex }} y^{k+1}=y^{1} .
$$


Since lexicographic order is a partial order, we infer $y^{1}=y^{2}=\cdots=y^{k}$, contradicting the assumption that $C$ is a cycle.

Moving on to the second case, suppose there exists $i, j$ with $z^{i} \neq z^{j}$. Then there exists $i \in[k]$ with $z^{i} \neq z^{i+1}$. Recall that since $\left(y^{i}, y^{i+1}\right) \in C, y^{i} \in B\left(y^{i+1}\right)$. Clearly, $y^{i+1} \in B\left(y^{i+1}\right)$. Note for all $x$ and $y$ such that $x \leq y$, and for all prices $p$ and budgets $b$, we have $y \in B(p, b)$ implies $x \in B(p, b)$. Since by construction $z^{i} \leq y^{i}$ and $z^{i+1} \leq y^{i+1}$, we infer $z^{i}, z^{i+1} \in B\left(y^{i+1}\right)$. From this fact, the definitions of $z^{i}$ and $z^{i+1}$, and the fact that distinct lattice points in $X$ have distinct ranks (as stated in Lemma 12), we can infer $r\left(z^{i}\right)<r\left(z^{i+1}\right)$.

Of course, for any $j$, if $z^{j}=z^{j+1}$ then $r\left(z^{j}\right)=r\left(z^{j+1}\right)$. Hence for all $j \in[k]$, either $r\left(z^{j}\right)=r\left(z^{j+1}\right)$ or $r\left(z^{j}\right)<r\left(z^{j+1}\right)$, so that for all $j \in[k], r\left(z^{j}\right) \leq r\left(z^{j+1}\right)$, and moreover there is at least one strict inequality: $r\left(z^{i}\right)<r\left(z^{i+1}\right)$. Altogether these facts yield

$$
r\left(z^{1}\right) \leq \cdots \leq r\left(z^{i}\right)<r\left(z^{i+1}\right) \leq \cdots \leq r\left(z^{k+1}\right)=r\left(z^{1}\right)
$$

contradicting the well-ordering of $\leq$ for $\mathbb{Z}_{++}$.

Corollary 15. A data $\left\{\left(x^{i}, p^{i}, b^{i}\right)\right\}_{i=0}^{n}$ is rationalizable if and only if algorithm $\mathcal{A}$ computes a demand function $\psi$ such that $\psi$ explains the data and is generated by a monotone utility function.

Proof. By Theorem 3, Lemma 10, and Theorem 14, a data $\left\{\left(x^{i}, p^{i}, b^{i}\right)\right\}_{i=0}^{n}$ is rationalizable if and only if the constructed $\psi$ is such that $\left(R_{\psi} \cup \leq\right)^{*}$ is acyclic. Acyclicity implies (by a version of Szpilrajn's lemma) that $\left(R_{\psi} \cup \leq\right)^{*}$ has an extension to $\mathbb{Z}_{+}^{d}$. Since $\mathbb{Z}_{+}^{d}$ is countable, the extension trivially satisfies the standard order-denseness condition for a utility representation. The utility representation rationalizes $\psi$ by definition of $R_{\psi}$; and it is monotone because it represents an extension of $\leq$.

\section{Appendix B Hardness Proofs}

In this section we prove various hardness and inapproximability results cited in Section 2 . These results show that the consumer is faced with solving an intractable problem according to a worst-case view of computational complexity in the consumer choice model. These results are not surprising or difficult but are included for completeness of presentation. ${ }^{8}$

We start by proving the hardness of maximizing utility subject to a budget constraint under monotone utility functions.

Lemma 16. Maximizing utility subject to a budget constraint is NP-hard for monotone utility functions, both for divisible and indivisible goods.

\footnotetext{
${ }^{8}$ If the reviewers are aware of previous work that has already proven these results, we will happily remove these proofs.
} 
Proof. For indivisible goods, the problem is NP-hard even for linear utility functions, since the problem

$$
\arg \max \left\{u \cdot x: p \cdot x \leq b, x \in \mathbb{Z}_{+}\right\}
$$

is precisely the UNBOUNDED KNAPSACK problem, a classic NP-hard problem [16].

For divisible goods, we may fix arbitrary nonnegative constants $v_{1}, \ldots, v_{d}$ and define the utility function as $u(x)=\sum_{i=1}^{d} v_{i} \mathbf{1}\left\{x_{i} \geq 1\right\}$, where $\mathbf{1}\{P\}$ is the indicator variable for predicate $P$, i.e., $1\{P\}=1$ if $P$ is true, and $1\{P\}=0$ otherwise. Note $u$ is monotone. The resulting problem,

$$
\arg \max \left\{u(x): p \cdot x \leq b, x \in \mathbb{R}_{+}\right\},
$$

is again equivalent to UNBOUNDED KNAPSACK. The less-approximable DENSEST- $k$-SUBGRAPH problem can likewise be encoded: given $G=(V, E)$ set $u(x)=\sum_{(u, v) \in E} \mathbf{1}\left\{x_{u} \geq 1\right.$ and $\left.x_{v} \geq 1\right\}$, $b=k$, and $p=\mathbf{1}$.

Next, We provide an inapproximability result for maximizing a monotone supermodular function $f$ subject to a cardinality constraint. A special case of this problem called QUADRATIC KNAPSACK has long been known to be NP-hard [20], via a simple reduction from DENSEST- $k$ SUBGRAPH.

Despite much effort, accurate bounds on the best approximation factor obtainable for DENSEST- $k$-SUBGRAPH by an efficient algorithm under reasonable complexity assumptions have eluded all attempts to date. We can, however, exploit a construction due to Khot to obtain the following result.

Theorem 17. Fix any constant $c \geq 1$, and let $\alpha(n)=2^{n^{c}}$. There is no polynomial time $\alpha(n)$ approximation algorithm for maximizing a monotone supermodular function $f$ subject to a cardinality constraint, unless NP has randomized subexponential time algorithms, i.e., unless $N P \subseteq \cap_{\epsilon>0} B P T I M E\left(2^{n^{\epsilon}}\right)$, where $n=\log _{2}|\operatorname{dom}(f)|$ is the size of the groundset $V$.

Proof. Fix a constant $d \geq 1$ and a DENSEST- $k$-SUBGRAPH input $(\mathrm{G}=(\mathrm{V}, \mathrm{E}), \mathrm{k})$, and define $f(S):=\left|E \cap\left(\begin{array}{c}S \\ 2\end{array}\right)\right|$ for a subset of vertices $S$. Let $\tau(x):=2^{x^{d}}$ and let $F(S):=\tau(f(S))$ where $\tau(x):=2^{x^{d}}$. It may be easily verified that $f$ is monotone supermodular, hence $g \circ f$ is as well for any monotone increasing and convex function $g$, such as $\tau$. Thus $F$ is monotone supermodular. For now, assume that there is a distribution $\mathcal{D}$ on DENSEST- $k$-SUBGRAPH instances such that no polynomial time algorithm can distinguish instances with $\max _{|S| \leq k} f(S) \leq x$ from those with $\max _{|S| \leq k} f(S) \geq \alpha x$, when they are drawn from $\mathcal{D}$. Then it must be hard to distinguish instances with $\max _{|S| \leq k} F(S) \leq 2^{x^{d}}$ and those with $\max _{|S| \leq k} F(S) \geq 2^{(\alpha x)^{d}}$, because they correspond to exactly the same distinction. Note also that all integers in the range of $F$ can be written with polynomial in $n$ bits, so the problem size (which we take to be $|V|+\log F(V)$, where $V$ is the ground set) increases only by a polynomial factor when going from $f$ to $F$. It follows that polynomial-time $\alpha$-inapproximability for the first problem implies polynomial-time $\beta$-inapproximability for the latter, with $\beta:=2^{(\alpha x)^{d}} / 2^{x^{d}}$. It remains to establish a lower bound on $\beta$. 
To lower bound $\beta$, it is useful to understand possible values for $x$ and $\alpha$. Khot [21] (c.f. section C.2) proves the existence of a distribution $\mathcal{D}$ with $x=\Omega(n)$ and $\alpha=(1+\epsilon)$, under the complexity-theoretic assumption NP $\nsubseteq \cap_{\epsilon>0}$ BPTIME $\left(2^{n^{\epsilon}}\right)$. Define constant $\delta>0$ so that $x=\delta n$ in Khot's hard input distribution. Then $\log _{2}(\beta)=(\delta n)^{d}\left((1+\epsilon)^{d}-1\right)$. Setting $d \geq \frac{1}{\log _{2}(1+\epsilon)}$ ensures $\log _{2}(\beta) \geq(\delta n)^{d}$. Hence we have an inapproximability factor of $2^{(\delta n)^{d}}$ for arbitrarily large constant $d$, which is equivalent to an inapproximability factor of $2^{n^{c}}$ for arbitrarily large constant $c$.

\section{Appendix C Proofs of space complexity and running time lower bounds}

We provide proofs for Propositions (6), (7), (8), and (9) in the reverse order of their appearance. We do so because we find it convenient to start with the proofs for general theories and then proceed to the proofs for the theory of the consumer.

of Proposition 9. Any $g_{\tau}$ that rationalizes data $D:=\left\{\left(i_{k}, o_{k}\right)\right\}_{k=1}^{n}$ must encode a function $h:\left\{i_{k}: 1 \leq k \leq n\right\} \rightarrow \mathcal{O}$ that maps $i_{k}$ to $o_{k}$ for all $k$, since $g_{\tau}\left(i_{k}\right)=o_{k}$ by the assumption that $g_{\tau}$ rationalizes $D$. Fix an input sequence $\left\{i_{k}\right\}_{k=1}^{n}$. There are $N:=\left|\mathcal{R}_{\mathcal{G}}\left(\left\{i_{k}\right\}_{k=1}^{n}\right)\right|$ possible functions $h$ that map the elements of $\left\{i_{k}\right\}_{k=1}^{n}$ to outputs such that the resulting sequence $\left\{\left(i_{k}, h\left(i_{k}\right)\right)\right\}_{k=1}^{n}$ is rationalizable. Hence $g_{\tau}$ encodes one of $N$ states, which requires at least $\log _{2} N$ bits in the worst-case. Since $\left\{i_{k}\right\}_{k=1}^{n}$ is an arbitrary input sequence of length $n$, we may maximize over such sequences to obtain the claimed worst-case bound.

of Proposition 8. Fix theory $\mathcal{G}$, and let

$$
N:=\max _{\left(i_{1}, \ldots, i_{n}\right)}\left|\mathcal{R}_{\mathcal{G}}\left(\left\{i_{k}\right\}_{k=1}^{n}\right)\right|
$$

Let $\left\{i_{k}\right\}_{k=1}^{n}$ be an input sequence achieving $\left|\mathcal{R}_{\mathcal{G}}\left(\left\{i_{k}\right\}_{k=1}^{n}\right)\right|=N$. Without loss of generality, we assume the inputs $i_{k}$ are distinct, otherwise we may remove duplicates to obtain a smaller input sequence $\left\{i_{k}^{\prime}\right\}_{k=1}^{n^{\prime}}$ with $\left|\mathcal{R}_{\mathcal{G}}\left(\left\{i_{k}^{\prime}\right\}_{k=1}^{n^{\prime}}\right)\right|=N$. Fix a RAM with wordsize $w=\Theta(\log n)$, and assume there is a deterministic algorithm $\mathcal{A}^{*}$ in this machine model which computes a function $f: \mathcal{D} \times \mathcal{I} \rightarrow \mathcal{O}$ in worst case time $t=t(|D|,|i|)$ such that $i \mapsto f(D, i)$ is a demand function rationalizing $D$. Fix a data set $D \in \mathcal{R}_{\mathcal{G}}\left(\left\{i_{k}\right\}_{k=1}^{n}\right)$, and define $o_{k}$ for $k \in[n]$ such that $D=\left\{\left(i_{k}, o_{k}\right)\right\}_{k=1}^{n}$. Since we may permute the data points $\left(i_{k}, o_{k}\right)$ in $D$ arbitrary, this implies that for any $i \in\left\{i_{k}\right\}_{k=1}^{n}$ this algorithm $\mathcal{A}^{*}$ can infer the value of $f(D, i)$ after reading any $q:=t\left\lceil\frac{w}{s}\right\rceil$ data points, where $s$ is the number of bits to encode a data point. By the distinctness of the inputs, $s \geq \log _{2}(n)$, so that $q=\mathcal{O}(t)$. Since $\mathcal{A}^{*}$ can infer the value of $f(D, i)$ for all $i \in\left\{i_{k}\right\}_{k=1}^{n}$ using the same $q$ data points, it can compute $D$ given $\left\{i_{k}\right\}_{k=1}^{n}$. Hence, $q w$ bits suffice to identify which element of $\mathcal{R}_{\mathcal{G}}\left(\left\{i_{k}\right\}_{k=1}^{n}\right)$ is the actual data set, which implies $q w \geq \log _{2} N$. Therefore, we obtain $\log _{2} N \leq q w=\mathcal{O}(t w)$, which may be rearranged to yield the claimed bound $t=\Omega\left(\frac{\log _{2} N}{w}\right)$. 
of Proposition 7. By Proposition 9, it suffices to exhibit a sequence $\left\{i_{k}\right\}_{k=1}^{n}$ such that $\log \left|\mathcal{R}_{\mathcal{G}}\left(\left\{i_{k}\right\}_{k=1}^{n}\right)\right|=$ $\Omega(n \log n)$, which we will now proceed to do.

Suppose we are in the indivisible goods case. Recall an input in this theory consists of a price vector $p$ and a budget $b$, and an output is a consumption bundle. We suppose $p^{k}=\mathbf{1}$ and $b^{k}=k$ for all $k \in[n]$. Next, we count the number of rationalizable data sets whose inputs consist of such $p^{k}$ and $b^{k}$, i.e., $N:=\left|\mathcal{R}_{\mathcal{G}}\left(\left\{i_{k}\right\}_{k=1}^{n}\right)\right|$ where $i_{k}:=\left(p^{k}, b^{k}\right)$ for all $k$.

We claim $N=(n+1)$ !. To show this, we show that for all $k$ with $1 \leq j<n$, for any choice of $x^{1}, \ldots, x^{j-1}$ such that $\left\{\left(x^{k}, p^{k}, b^{k}\right)\right\}_{k=0}^{j-1}$ is rationalizable, there are $j+1$ choices for $x^{j}$ such that $\left\{\left(x^{i}, p^{i}, b^{i}\right)\right\}_{i=0}^{j}$ is also rationalizable. So, we proceed by induction on $j$. The base case, $j=1$ is trivial, since the consumer can buy one unit of either of the two goods. For the induction case, we claim that any consumption bundle $\left(x_{1}, x_{2}\right) \in \mathbb{Z}_{+}^{2}$ such that $x_{1}+x_{2}=j$ yields a rationalizable extension of the data. By Theorem 3, it suffices to prove that the new data point $\left(x^{j}, p^{j}, b^{j}\right):=\left(\left(x_{1}, x_{2}\right), \mathbf{1}, j\right)$ does not introduce a cycle into the induced preference relation $R_{0}$. However this is clearly impossible since $\left(x^{j}, x^{i}\right) \notin R_{0}$ for all $i<j$. Since the number of solutions $\left(x_{1}, x_{2}\right) \in \mathbb{Z}_{+}^{2}$ to $x_{1}+x_{2}=j$ is $j+1$, this concludes the induction step. Hence we get the rather trivial recurrence $N(j)=(j+1) N(j-1)$ with $N(1)=2$, which has solution $N:=N(n)=(n+1)$ !. Finally, we note that $\log _{2}(n+1) !=(1-o(1)) n \log _{2} n$, which completes the proof.

of Proposition 6. Proposition 6 claims that any function $f: \mathcal{D} \times \mathcal{I} \rightarrow \mathcal{O}$ such that $i \mapsto f(D, i)$ is a demand function rationalizing $D$ implies $f$ takes worst-case $\Omega(|D|)$ time, where $|D|$ is the number of data points (i.e., pairs $\left(i_{k}, o_{k}\right)$ ) in $D$. At a high level is this due to the simple observation that $f$ must read all of $D$ in the worst case. We can prove it by applying Proposition 8, using the construction of Proposition 7 which gives an input sequence with $\log \left|\mathcal{R}_{\mathcal{G}}\left(\left\{i_{k}\right\}_{k=1}^{n}\right)\right|=\Omega(n \log n)$. Combining the two immediately yields the claimed lower bound of $\Omega(n)$. 